\title{
Calendar and cycling ageing combination of batteries in electric vehicles
}

$\underline{\text { Eduardo REDONDO-IGLESIAS }}^{1}{ }^{1}$, Pascal VENET ${ }^{2}$, Serge PELISSIER $^{1}$

${ }^{1}$ IFSTTAR - LTE

${ }^{2}$ AMPERE UMR CNRS 5005

Université de Lyon, France

ESREF2018

Aalborg - 3 October 2018 
Calendar and cycling ageing combination of batteries in electric vehicles Outline

\section{Introduction}

\section{Accelerated Ageing Tests}

3. Analysis of results

4. Conclusion 


\section{Introduction}

- Electric vehicles

- Climate change and local pollution

- Higher energy efficiency

- Can help renewables development (smart grid)

- Battery is the limiting factor: cost, lifetime $\Rightarrow$ limited EV range

- Battery degradation

- Relies on ageing mechanisms (physico-chemical reactions)

- Calendar ageing: degradation during rest time

- Cycling ageing: degradation (directly) induced by charges and discharges

- Accelerated ageing tests

- What is the lifetime of a battery for a given application?

- How to optimise sizing and energy management of a battery pack for a longer life?

$\Rightarrow$ Get an answer to these questions in a short time! 


\section{Accelerated Ageing Tests}

Electric vehicle use profile

- Domestic use

- Small electric car

- Battery pack: 16kWh (320V x 50Ah)

- Daily trip (home-work-home)

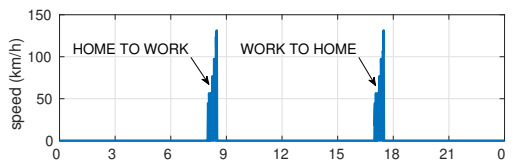

- example: WLTC (single trip)

- 30 minutes

- $23 \mathrm{~km}$
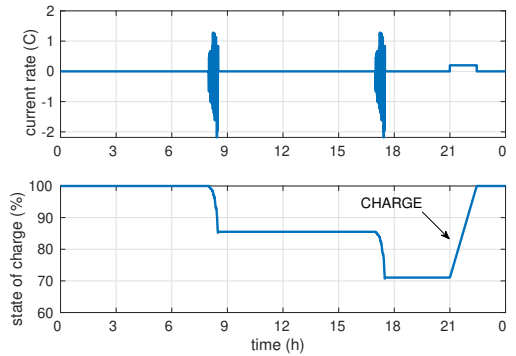


\section{Accelerated Ageing Tests}

Electric vehicle use profile

- Domestic use

- Small electric car

- Battery pack: 16kWh (320V x 50Ah)

- Daily trip (home-work-home)

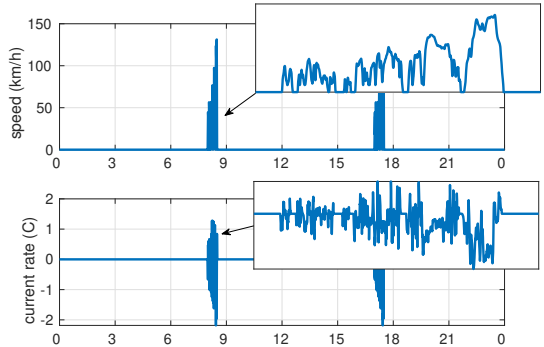

- example: WLTC (single trip)

- 30 minutes

- $23 \mathrm{~km}$

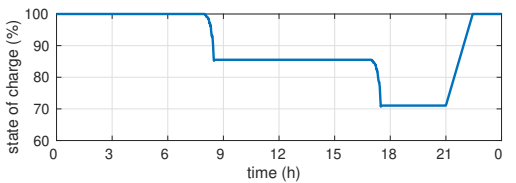


Accelerated Ageing Tests

Electric vehicle use profile

- Battery use

- during each trip

$\Delta S o C \sim 15 \%$

- $I_{\max }=2 C, I_{\text {avg }}=C / 3$

- duration $\sim 0.5$ hours

- charge

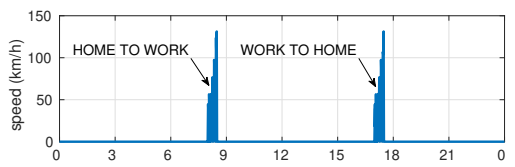

$\Delta S o C \sim 30 \%$

$I_{c s t}=C / 5$

duration $\sim 1.5$ hours

- rest periods

$I=0$

$\sim 21.5$ hours a day
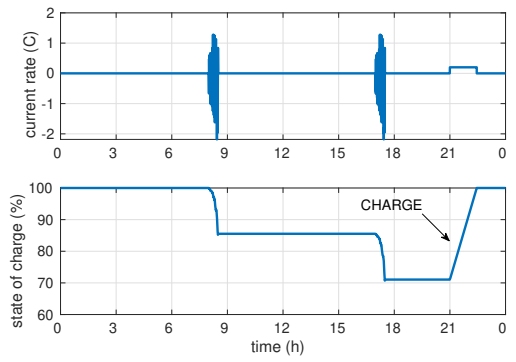

* $1 \mathrm{C}=50 \mathrm{~A}$ ( $50 \mathrm{Ah}$ battery pack) 
Accelerated Ageing Tests

Electric vehicle use profile

- Battery use

- during each trip

$\triangle S o C \sim 15 \%$

- $I_{\max }=2 C, I_{\text {avg }}=C / 3$

duration $\sim 0.5$ hours

$\checkmark$ charge

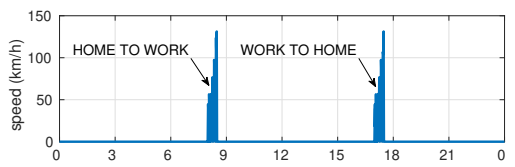

$\Delta S o C \sim 30 \%$

$I_{c S t}=C / 5$

duration $\sim 1.5$ hours

- rest periods

$I=0$

$\sim 21.5$ hours a day

Battery is at rest $90 \%$ of time
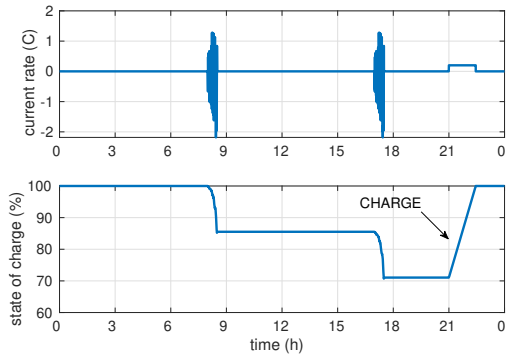

$* 1 \mathrm{C}=50 \mathrm{~A}(50 \mathrm{Ah}$ battery pack) 
Accelerated Ageing Tests

Electric vehicle use profile

- Battery use

$\checkmark$ during each trip

$\Delta$ - $\triangle$ oC $\sim 15 \%$

$I_{\max }=2 C, I_{\text {avg }}=C / 3$

- duration $\sim 0.5$ hours

$\checkmark$ charge

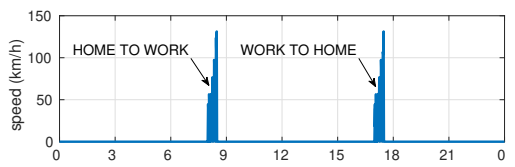

$\Delta S o C \sim 30 \%$

$I_{c S t}=C / 5$

duration $\sim 1.5$ hours

- rest periods

$I=0$

$\sim 21.5$ hours a day

Battery is at rest $90 \%$ of time
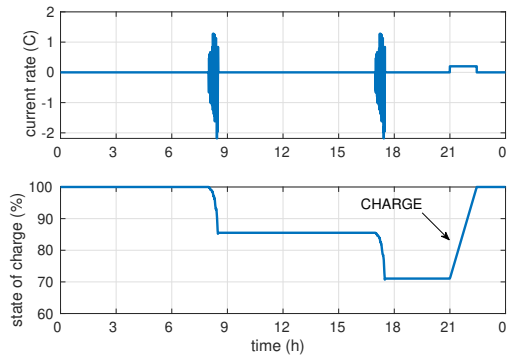

* $1 \mathrm{C}=50 \mathrm{~A}(50 \mathrm{Ah}$ battery pack)

- Is cycling ageing negligible respect to calendar ageing?

Combined ageing $\simeq$ Calendar ageing ? 
Accelerated Ageing Tests

Electric vehicle use profile

- Battery use

- during each trip

$\Delta$ - $\triangle$ oC $\sim 15 \%$

$I_{\max }=2 C, I_{\text {avg }}=C / 3$

duration $\sim 0.5$ hours

$\checkmark$ charge

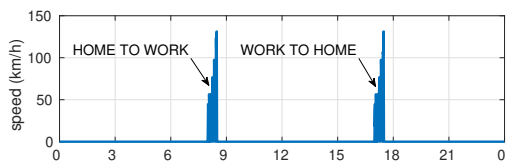

$\Delta S o C \sim 30 \%$

$I_{c s t}=C / 5$

duration $\sim 1.5$ hours

- rest periods

$I=0$

$\sim 21.5$ hours a day

Battery is at rest $90 \%$ of time
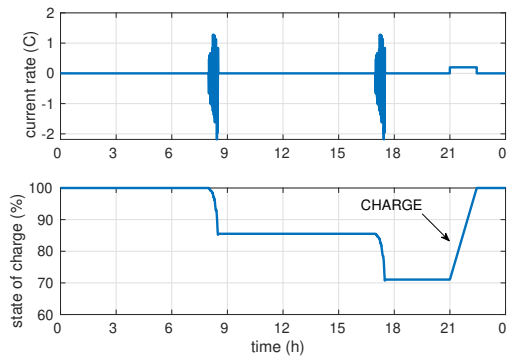

* $1 \mathrm{C}=50 \mathrm{~A}(50 \mathrm{Ah}$ battery pack)

- Is cycling ageing negligible respect to calendar ageing?

Combined ageing $\simeq$ Calendar ageing ?

- Are both ageing modes arithmetically cumulative?

- Combined ageing $\simeq$ Calendar ageing + Cycling ageing 
Accelerated Ageing Tests

Experimental setup

- Cell technology:

- lithium-ion NMC/graphite (3.7 V) 
Accelerated Ageing Tests

Experimental setup

- Cell technology:

- lithium-ion NMC/graphite (3.7 V)

- Calendar ageing

- $\mathrm{SoC}=100,90,80,70,50 \%$ 
Accelerated Ageing Tests

Experimental setup

- Cell technology:

- lithium-ion NMC/graphite (3.7 V)

- Calendar ageing

- $\mathrm{SoC}=100,90,80,70,50 \%$

- Cycling ageing

- 7 different profiles

- most of time at rest

- very low current rate:

- $\mathrm{C} / 2$ discharge
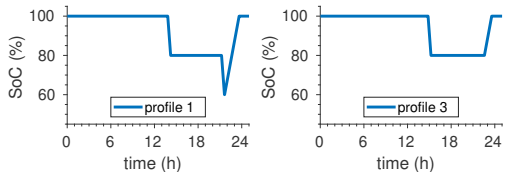

- $\mathrm{C} / 5$ charge
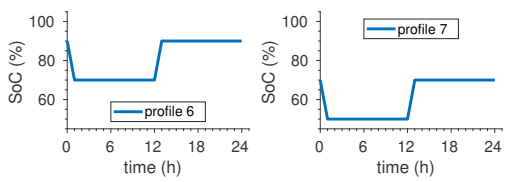


\section{Accelerated Ageing Tests}

\section{Experimental setup}

- Cell technology:

lithium-ion NMC/graphite (3.7 V)

- Calendar ageing

- $\mathrm{SoC}=100,90,80,70,50 \%$

- Cycling ageing

- 7 different profiles

- most of time at rest

- very low current rate:

- $\mathrm{C} / 2$ discharge
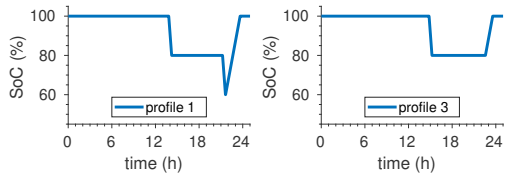

- C/5 charge
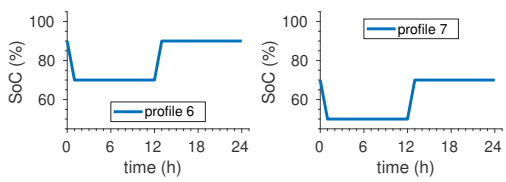

- For all ageing tests (cycling + calendar)

- $\mathrm{T}=60^{\circ} \mathrm{C}$

- 3 cells for each ageing condition ( 15 cells in calendar ageing, 21 cells in cycling ageing)

- capacity measurements every month 


\section{Accelerated Ageing Tests}

Results

\section{Calendar ageing}

- vs. time:

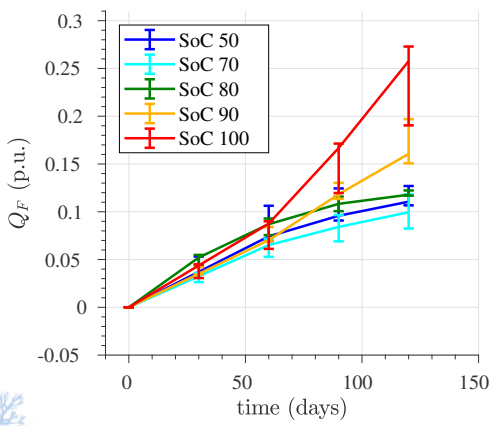

- vs. SoC (120 days):

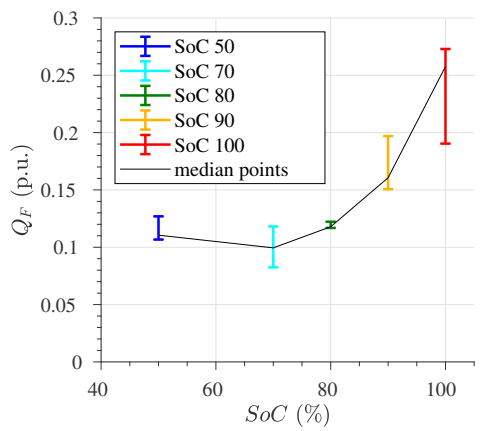

\section{very non linear with SoC}




\section{Accelerated Ageing Tests}

Results

\section{Cycling ageing}


faster degradations when charging to $100 \%$ 
Analysis of results

\section{$\underline{\text { How to separate calendar and cycling parts of ageing? }}$}


Analysis of results

\section{How to separate calendar and cycling parts of ageing?}

- Cumulative damage approach 
Analysis of results

\section{How to separate calendar and cycling parts of ageing?}

- Cumulative damage approach

- Lifetime modelling of devices 
Analysis of results

\section{How to separate calendar and cycling parts of ageing?}

- Cumulative damage approach

- Lifetime modelling of devices

- Decompose device's life into «events» 
Analysis of results

How to separate calendar and cycling parts of ageing?

- Cumulative damage approach

- Lifetime modelling of devices

- Decompose device's life into «events»

$$
Q_{F}=Q_{F, c a l}+Q_{F, c y c}
$$

- $Q_{F}$ : Total capacity fade

- $Q_{F, \text { cal }}$ : Capacity fade due to calendar ageing

- $Q_{F, \text { cyc }}$ : Capacity fade caused by cycling 
Analysis of results

Cumulative damage approach

Cycling tests:

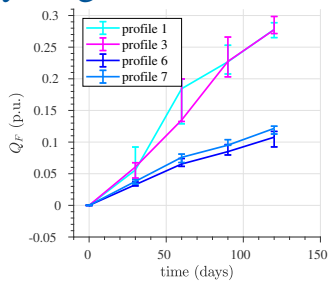

\section{Calendar tests:}

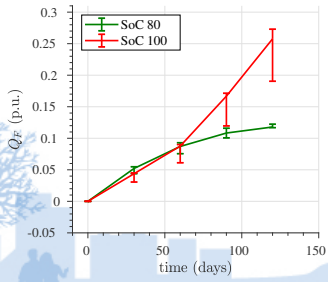

Example: profile 3 


\section{Analysis of results}

Cumulative damage approach

\section{Cycling tests:}

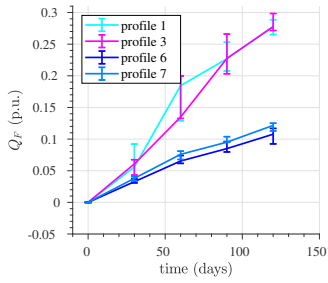

\section{Calendar tests:}

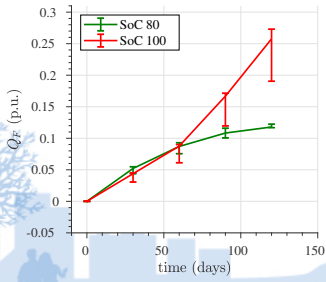

Example: profile 3

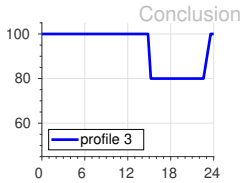

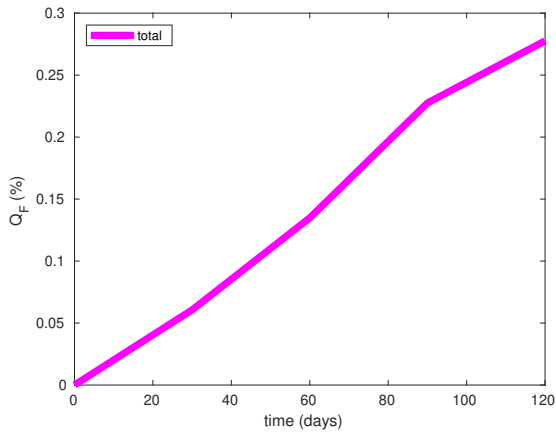

1. $Q_{F}$ : measured capacity fade 
Analysis of results

Cumulative damage approach

Cycling tests:

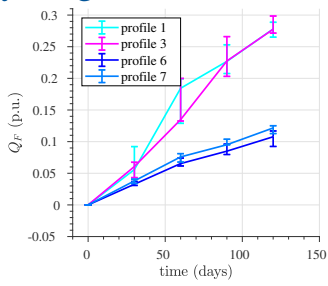

\section{Calendar tests:}

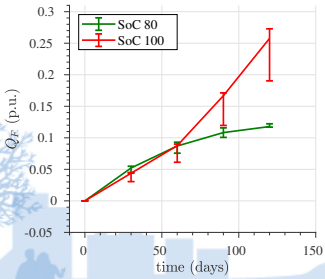

Example: profile 3

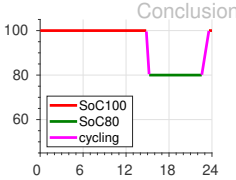

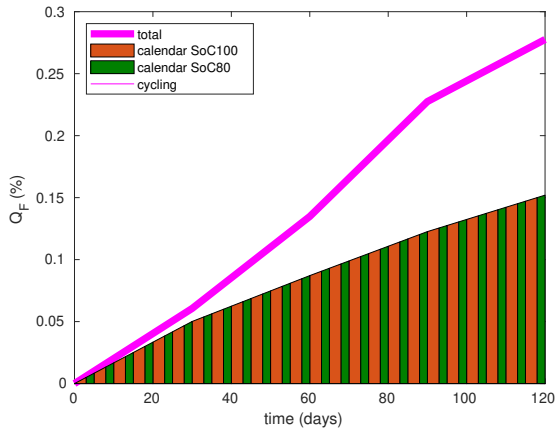

1. $Q_{F}$ : measured capacity fade

2. $Q_{F, \text { cal }}$ : from calendar ageing tests 
Analysis of results

Cumulative damage approach

Cycling tests:

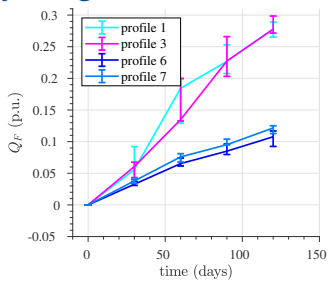

Calendar tests:

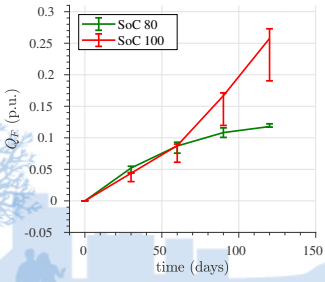

Example: profile 3

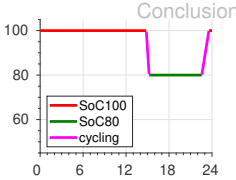

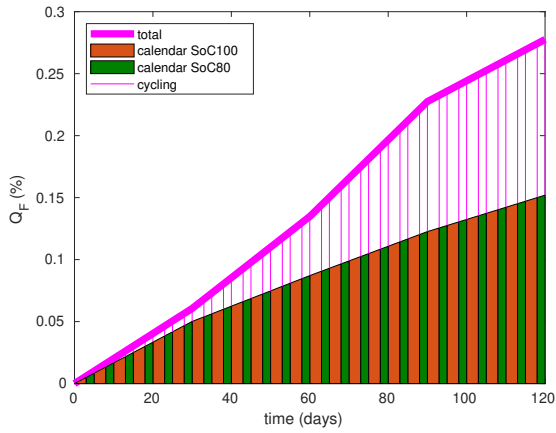

1. $Q_{F}$ : measured capacity fade

2. $Q_{F, \text { cal }}$ : from calendar ageing tests

3. $Q_{F, \text { cyc }}$ : obtained by difference 


\section{Analysis of results}

Calendar and cycling parts of ageing

Capacity fade per cycle:

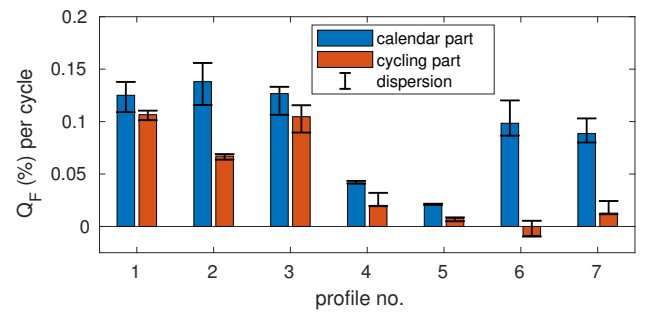


Analysis of results

Calendar and cycling parts of ageing

Capacity fade per cycle:

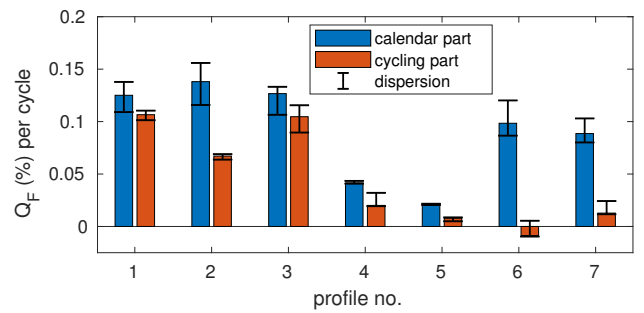

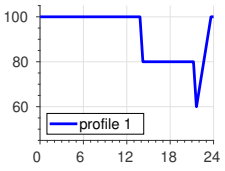

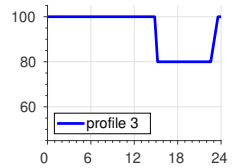

- Profile 1 vs. 3: $Q_{F, c y c} \neq f($ cycled $A h)$ 
Analysis of results

Calendar and cycling parts of ageing

Capacity fade per cycle:



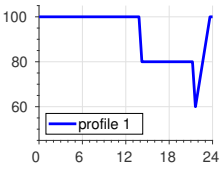
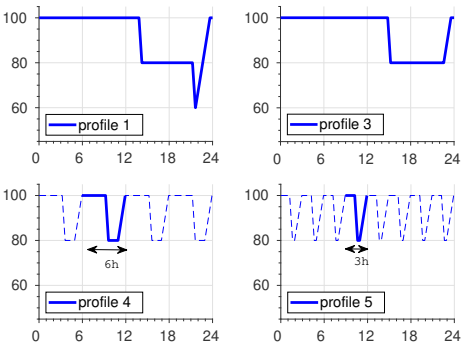

- Profile 1 vs. 3: $Q_{F, c y c} \neq f($ cycled $A h)$

- Profiles 3, 4 and 5: frequency $\uparrow \Rightarrow Q_{F, c y c} \downarrow$ 
Analysis of results

Calendar and cycling parts of ageing

Capacity fade per cycle:

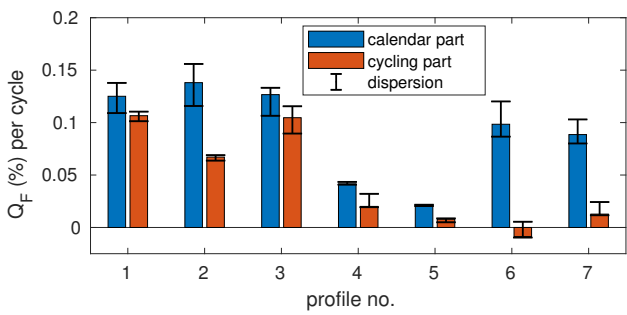

- Profile 1 vs. 3: $Q_{F, c y c} \neq f($ cycled $A h)$

- Profiles 3, 4 and 5: frequency $\uparrow \Rightarrow Q_{F, c y c} \downarrow$

- Profiles 6 and 7: lower SoCs $\Rightarrow Q_{F, c y c} \simeq 0$
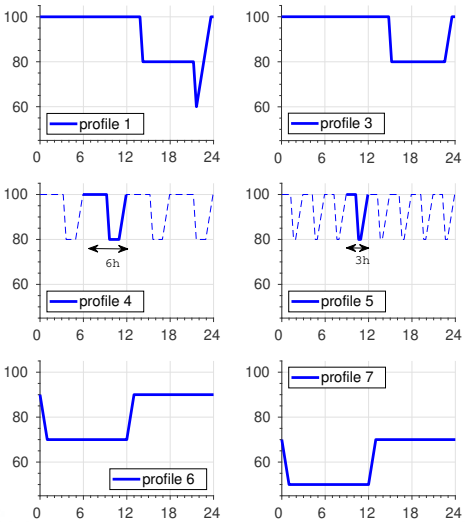


\section{Conclusions:}

- Electric vehicle application

not continuous cycling (calendar and cycling combination)

- very low current rates

- Calendar ageing is very non linear respect to SoC

- Cycling ageing combined to calendar ageing may accelerate degradation

- very significant at full charges $(100 \%)$

- less influence when charging at lower SoC levels (90, 70\%)

- decreases rapidly with cycling frequency (from 24 to 6 or 3 hours) 


\section{Conclusions:}

- Electric vehicle application

not continuous cycling (calendar and cycling combination)

- very low current rates

- Calendar ageing is very non linear respect to SoC

- Cycling ageing combined to calendar ageing may accelerate degradation

- very significant at full charges $(100 \%)$

- less influence when charging at lower SoC levels (90, 70\%)

- decreases rapidly with cycling frequency (from 24 to 6 or 3 hours)

$\Rightarrow$ Is cycling ageing negligible respect to calendar ageing?

- Not when charging to $100 \%$ SoC. 


\section{Conclusions:}

- Electric vehicle application

not continuous cycling (calendar and cycling combination)

- very low current rates

- Calendar ageing is very non linear respect to $\mathrm{SoC}$

- Cycling ageing combined to calendar ageing may accelerate degradation

- very significant at full charges (100\%)

- less influence when charging at lower SoC levels (90, 70\%)

- decreases rapidly with cycling frequency (from 24 to 6 or 3 hours)

$\Rightarrow$ Is cycling ageing negligible respect to calendar ageing?

- Not when charging to $100 \%$ SoC.

$\Rightarrow$ Are both ageing modes arithmetically cumulative?

No, it depends of SoC, frequency and shape of cycles. 


\section{Conclusions:}

- Electric vehicle application

not continuous cycling (calendar and cycling combination)

- very low current rates

- Calendar ageing is very non linear respect to $\mathrm{SoC}$

- Cycling ageing combined to calendar ageing may accelerate degradation

- very significant at full charges (100\%)

- less influence when charging at lower SoC levels (90, 70\%)

- decreases rapidly with cycling frequency (from 24 to 6 or 3 hours)

$\Rightarrow$ Is cycling ageing negligible respect to calendar ageing?

- Not when charging to $100 \%$ SoC.

$\Rightarrow$ Are both ageing modes arithmetically cumulative?

No, it depends of SoC, frequency and shape of cycles.

\section{Further work:}

- Incremental capacity analyses (ageing mechanisms)

- Empirical degradation model (single or multiple ageing mechanism)

- Validation tests 


\section{Thank you for your attention Any questions?}

Eduardo REDONDO-IGLESIAS

cv. archives-ouvertes.fr/redondo


Analysis of results

Calendar and cycling parts of ageing (profile 1 vs. 2)

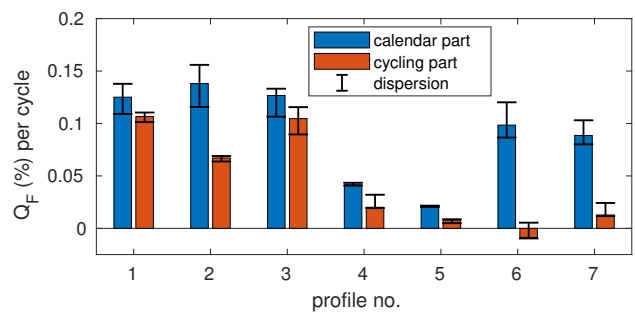

CCCV charge protocol:

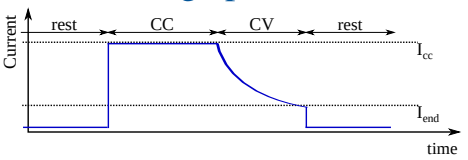

- Profile 2 is like profile 1 but:

- final charge with CC 6 days/week

- final charge with CCCV 1 day/week

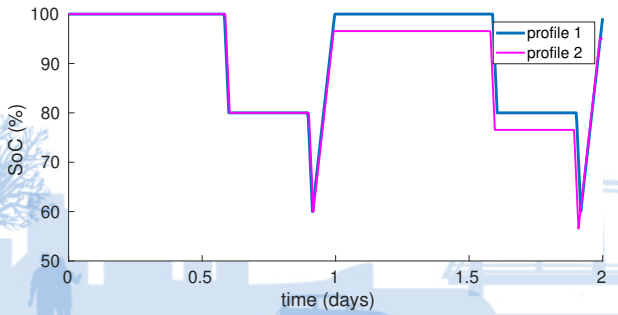


Analysis of results

Calendar and cycling parts of ageing (profile 1 vs. 2)

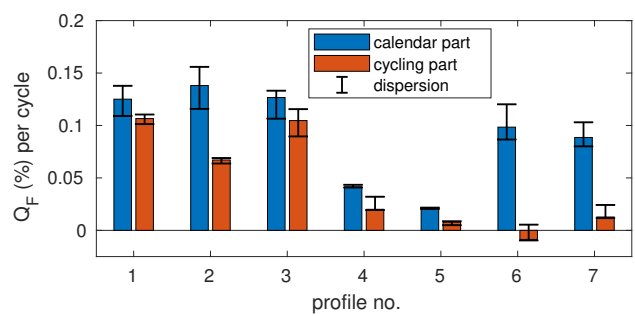

CCCV charge protocol:

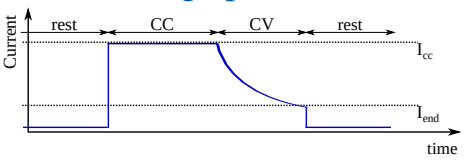

- Profile 2 is like profile 1 but:

- final charge with CC 6 days/week

- final charge with CCCV 1 day/week

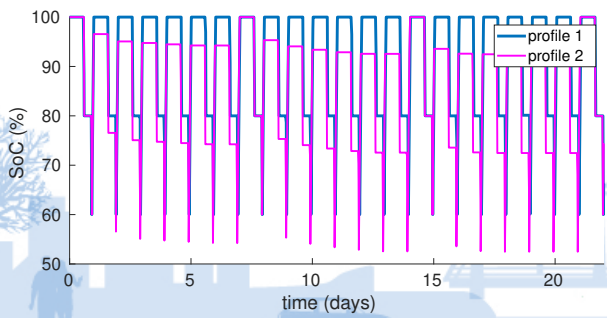


Analysis of results

Calendar and cycling parts of ageing (profile 1 vs. 2)

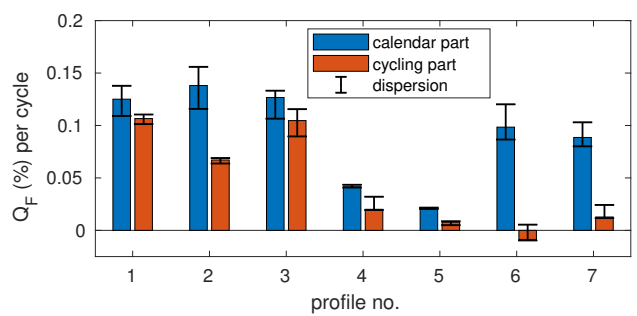

CCCV charge protocol:

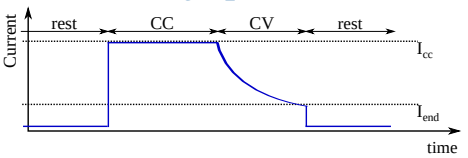

- Profile 2 is like profile 1 but:

- final charge with CC 6 days/week

- final charge with CCCV 1 day/week

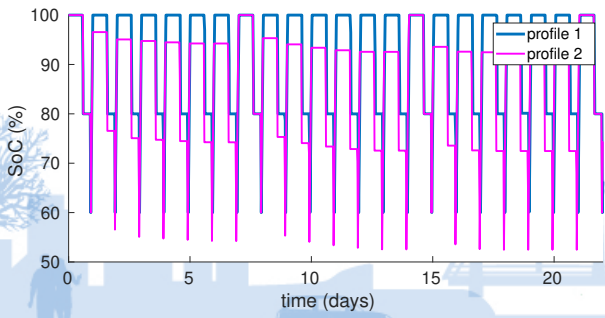

\section{Profile 1 vs. profile 2:}

more $\mathrm{CV}$ phases $\Rightarrow Q_{F, c y c} \uparrow$ 